\section{Lipofuscin, lipofuscin-like pigments and autofluorescence}

\author{
G. Di Guardo \\ Faculty of Veterinary Medicine, \\ University of Teramo, Italy
}

\section{Abstract}

A brief overview is here provided on lipofuscin and lipofuscin-like substances, with particular reference to their biological significance as well as to their cellular origin and pathophysiological role. Special emphasis is also placed on the mutual relationships between lipofuscin and lipofuscin-like lipopigments on one side, and cell autofluorescence on the other.

\section{Introduction}

I have read with great interest the outstanding review article by Croce and Bottiroli on autofluorescence (AF) spectroscopy and imaging, recently published in European Journal of Histochemistry. ${ }^{1}$ In this respect, as correctly stated by the aforementioned authors, the frequent presence of endogenous biomolecules with a fluorophore-like behavior in organisms and living systems across the entire life kingdom makes AF a commonly occurring biological phenomenon. As still correctly highlighted by the authors, several endogenous fluorophores - such as porphyrins, a variety of vitamins (vitamin A, riboflavin, thiamine), structural proteins, lipofuscin and ceroid pigments, along with others - may be responsible for cell and tissue $\mathrm{AF}^{1}$. Noteworthy, intracellular $\mathrm{AF}$ has been recently characterized as a selective biomarker for epithelial cancer stem cells (CSCs), with such epithelial CSC-specific AF being also accompanied by a strongly invasive and chemoresistant cell phenotype. ${ }^{2}$ After having excluded autophagy, riboflavin (vitamin $B_{2}$ ) has been recognized as the biomolecular substrate responsible for the AF selectively observed in epithelial CSCs ${ }^{2}$. Surprisingly enough, however, lipofuscin and lipofuscinlike compounds/lipopigments were not included among the potential-putative factors responsible, at least in part, for the $\mathrm{AF}$ seen in epithelial CSCs. On the basis of what above, while it should be duly highlighted, on one side, that almost no doubts exist on the absolute relevance, biological significance and extremely important implications (also from the fight against cancer standpoint) of the original and intriguing results of the aforesaid study, ${ }^{2}$ it should be additionally emphasized, on the other side, that not considering lipofuscin and lipofuscin-like compounds among the biomolecular substrates potentially-putatively responsible for the AF specifically detected in epithelial CSCs could be regarded as a conceptual error leading to a methodological slip.

\section{Autofluorescent lipofuscin and lipofuscin-like pigments}

Indeed, alongside with riboflavin (vitamin $B_{2}$ ) and among the several previously mentioned endogenous biomolecules exhibiting a fluorophore-like activity, ${ }^{1}$ autofluorescent lipofuscin and lipofuscin-like pigments may also occur in the cell cytoplasmic compartment, with these heterogeneous substances also showing variability in their protein, lipid and/or carotenoid composition, crosslinks and oxidation level. ${ }^{3}$ Furthermore, the formation of lipofuscin and lipofuscin-like compounds is known to take place under a variety of pathophysiological conditions, such as oxidative stress and ageing, ${ }^{4,5}$ two sides of the same coin which are essentially characterized by a progressive unbalance between protein damage and clearance, leading at its turn to an increased protein homeostasis disturbance, with accumulation of oxidized proteins' aggregates and, subsequently, of highly-cross linked materials such as lipofuscin and lipofuscinlike lipopigments, affecting cell viability. Cell viability appears to be especially compromised in postmitotic cells, such as neurons, provided they cannot reduce their lipofuscin load through karyokinesis. ${ }^{4}$ At the light of what above, lipofuscin and lipofuscin-like compounds should be regarded as aggregates of undigested cell materials resulting from phagocytosis and autophagy processes and accumulating as endocytoplasmic granules under both physiological and pathological conditions. ${ }^{4,5}$ During ageing, for instance, lipofuscin accumulation may be physiologically observed in the liver as well as in the central nervous system, at the level of which the occurrence of lipofuscin, lipofuscin-like lipopigments and ceroid may be also detected during oxidative stress, or as a response to given lysosomal storage diseases (Batten disease or neuronal ceroid lipofuscinosis $)^{6}$ and to a number of physical and chemical noxae, such as radiation, cisplatin, lead $(\mathrm{Pb})$ and mercury (methyl$\mathrm{Hg}$ ), under both natural and experimental disease conditions..$^{17-10}$ Moreover, lipofuscin and lipofuscin-like substances, which alongside with their well known AF properties ${ }^{1,11}$ may be easily demonstrated in host cells by means of Schmorl and/or Sudan Black histochemical stains, ${ }^{10,12,13}$ have been reported to increase in human mesenchymal stem cells subjected to
Correspondence: Prof. Giovanni Di Guardo, DVM, Dipl. ECVP, Faculty of Veterinary Medicine, University of Teramo, piazza Aldo Moro 45, 64100 Teramo, Italy.

Tel. +39.861.266933 - Fax: +39.861. 266865 .

E-mail: gdiguardo@unite.it

Key words: Lipofuscin, lipofuscin-like pigments, ceroid, ceroid lipofuscinosis, autofluorescence, fluorescence microscopy.

Received for publication: 10 January 2015 . Accepted for publication: 27 January 2015.

This work is licensed under a Creative Commons Attribution NonCommercial 3.0 License (CC BYNC 3.0).

(C) Copyright G. Di Guardo, 2015

Licensee PAGEPress, Italy

European Journal of Histochemistry 2015; 59:2485 doi:10.4081/ejh.2015.2485

oxidative stress, ${ }^{14}$ while decreasing in murine embryonic stem cells undergoing differentiation, ${ }^{15}$ a finding that might be of relevance also in relation to the recently described epithelial CSC-specific AF. ${ }^{2}$ Beside what above, lipofuscin bodies may be observed in a number of additional pathologic conditions, both neoplastic and non-neoplastic, such as pancreatic tumours, non-choroidal melanomas, mammary gland carcinomas, pigmented eyelid cysts, retinal degenerative processes like age-related macular degeneration and brown bowel syndrome or intestinal lipofuscinosis. ${ }^{12,13,16,17}$

As far as the biological effects exerted by lipofuscin and lipofuscin-like lipopigments in host cells are concerned, they have not been precisely determined, thus far. Nevertheless, beside being a powerful oxidants' source, lipofuscin has also been shown to incorporate iron in a redox-active fashion and to induce apoptosis, with the degree of lipofuscin-driven cell damage and cytotoxicity appearing to be tightly related to the iron concentration present in lipofuscin itself. ${ }^{18}$

Finally, regarding the fate and processing of lipofuscin and lipofuscin-like substances inside host cells, it should be also underscored that, as in the case of many other large protein aggregates, the process responsible for their uptake and degradation is macroautophagy, the best characterized among autophagy-related pathways. ${ }^{19}$ The first step of macroautophagy consists in the formation of the socalled autophagosome, a double membranebound vesicle engulfing the aforementioned protein aggregates, so that they can be subsequently delivered to the cell endo-lysosomal system for digestion, with the resulting structure being named autophagolysosome. ${ }^{4}$ In this respect, while macroautophagy has been 
recently found to be impaired in ageing by endocellular lipofuscin accumulation, ${ }^{20}$ additional experimental data obtained in aged mice support the hypothesis that lysosomal acidification could prevent the accumulation of lipofuscin and lipofuscin-like lipopigments in retinal pigmented epithelial cells, which are consistently damaged in human age-related macular degeneration. ${ }^{21}$ Notwithstanding what above, another recent study suggests that autophagosomes and lysosomes are not compulsorily involved in lipofuscin formation but they rather act as a storage site for biomolecular aggregates of this kind, thereby contributing to mitigate their cytotoxicity. ${ }^{22}$

\section{Concluding remarks}

Although the first microscopic evidence of AF was reported over 100 years ago, ${ }^{1}$ the interest of the scientific community in this widespread biological phenomenon continues to be remarkable, as clearly exemplified, among others, by the recent characterization of $\mathrm{AF}$ as a biomarker for epithelial CSCs. ${ }^{2}$ In this respect, few doubts also exist about the enormous possibilities offered by the increasingly sophisticated biomolecular and microscopic techniques for a better characterization of autofluorescent lipofuscin and lipofuscin-like lipopigments, with special reference to their biological significance, cell origin and fate, along with their pathophysiological role in health, ageing and disease.

\section{References}

1. Croce AC, Bottiroli G. Autofluorescence spectroscopy and imaging: a tool for biomedical research and diagnosis. Eur $\mathrm{J}$ Histochem 2014;58:2461.

2. Miranda-Lorenzo I, Dorado J, Lonardo E, Alcala S, Serrano AG, Clausell-Tormos J, et al. Intracellular autofluorescence: a biomarker for epithelial cancer stem cells. Nat Methods 2014;11:1161-9.
3. Wolman M. Lipid pigments (chromolipids): their origin, nature, and significance. Pathobiol Annu 1980;10:253-67.

4. Höhn A, Grune T. Lipofuscin: formation, effects and role of macroautophagy. Redox Biol 2013;1:140-4.

5. Rattan SI, Keeler KD, Buchanan JH, Holliday R. Autofluorescence as an index of ageing in human fibroblasts in culture. Biosci Rep 1982;2:561-7.

6. Palmer DN, Oswald MJ, Westlake VJ, Kay GW. The origin of fluorescence in the neuronal ceroid lipofuscinoses (Batten disease) and neuron cultures from affected sheep for studies of neurodegeneration. Arch Gerontol Geriatr 2002;34:343-57.

7. Fonnum F, Lock EA. Cerebellum as a target for toxic substances. Toxicol Lett 2000;112113:9-16.

8. Croce AC, Pisu MB, Roda E, Avella D, Bernocchi G, Bottiroli G. Autofluorescence properties of rat cerebellum cortex during postnatal development. Lasers Surg Med 2006;38:598-607.

9. Patková J, Vojtíšek M, T ma J, Vožeh F, Knotková J, Santorová P, et al. Evaluation of lipofuscin-like pigments as an index of lead-induced oxidative damage in the brain. Exp Toxicol Pathol 2012;64:51-6.

10. Mazzariol S, Di Guardo G, Petrella A, Marsili L, Fossi MC, Leonzio C, et al. Sometimes sperm whales (Physeter macrocephalus) cannot find their way back to the high seas: a multidisciplinary study on a mass stranding. PLoS One 2011;6:e19417.

11. Armstrong D, Wilhelm J, Smid F, Elleder M. Chromatography and spectrofluorometry of brain fluorophores in neuronal ceroid lipofuscinosis (NCL). Mech Ageing Dev 1992;64:293-302.

12. Daum 0, Sima R, Mukensnabl P, Vanecek T, Brouckova M, Benes Z, et al. Pigmented solid-pseudopapillary neoplasm of the pancreas. Pathol Int 2005;55:280-4.

13. Penouil MH, Gourhant JY, Segretin C, Weedon D, Rosendahl C. Non-choroidal yellow melanoma showing positive staining with Sudan Black consistent with the presence of lipofuscin: a case report.
Dermatol Pract Concept 2014;4:45-9.

14. Rice WL, Kaplan DL, Georgakoudi I. Twophoton microscopy for non-invasive, quantitative monitoring of stem cell differentiation. PLoS One 2010;5:e10075.

15. Santin G, Paulis M, Vezzoni P, Pacchiana G, Bottiroli G, Croce AC. Autofluorescence properties of murine embryonic stem cells during spontaneous differentiation phases. Lasers Surg Med 2013;45:597-607.

16. Donatello S, Hudson L, Cottell DC, Blanco A, Aurrekoetxea I, Shelly MJ, et al. An imbalance in progenitor cell populations reflects tumour progression in breast cancer primary culture models. J Exp Clin Cancer Res 2011;30:45.

17. Białas M, Demczuk S, Dyduch G, Drabik G, Chrupek M, Oko K. Brown bowel syndrome (intestinal lipofuscinosis) - a case report and review of the literature. Pol J Pathol 2013;64:228-31.

18. Höhn A, Jung T, Grimm S, Grune T. Lipofuscin-bound iron is a major intracellular source of oxidants: role in senescent cells. Free Radic Biol Med 2010;48:1100-8.

19. Codogno P. Shining light on autophagy. Nat Rev Mol Cell Biol 2014;15:153.

20. Ureshino RP, Rocha KK, Lopes GS, Bincoletto C, Smaili SS. Calcium signaling alterations, oxidative stress, and autophagy in aging. Antioxid Redox Signal 2014;21:123-37.

21. Guha S, Liu J, Baltazar G, Laties AM, Mitchell CH. Rescue of compromised lysosomes enhances degradation of photoreceptor outer segments and reduces lipofuscin-like autofluorescence in retinal pigmented epithelial cells. Adv Exp Med Biol 2014;801:105-11.

22. Höhn A, Sittig A, Jung T, Grimm S, Grune T. Lipofuscin is formed independently of macroautophagy and lysosomal activity in stress-induced prematurely senescent human fibroblasts. Free Radic Biol Med 2012;53:1760-9. 